\title{
Werner syndrome: quantitative assessment of skin aging
}

\section{Vittorio Mazzarello \\ Marco Ferrari \\ Pasquale Ena}

Skinlab, Department of Biomedical Sciences, University of Sassari, Sassari, Italy
Correspondence: Vittorio Mazzarello Skinlab, Department of Biomedical Sciences, University of Sassari, Viale San Pietro 43, 07I00 Sassari, Italy

Tel +3979228536

Fax +39792 28520

Email vmazza@uniss.it
This article was published in the following Dove Press journal: Clinical, Cosmetic and Investigational Dermatology

Background: Werner syndrome (WS) is a rare autosomal recessive disorder characterized by premature aging in adults. Although not sufficient to diagnose WS, persistent short stature and alteration of the dentition are among the few early signs that appear at puberty and can lead to a suspected diagnosis.

Objective: The study aimed at quantifying the signs of WS skin aging through biophysical parameters to find new parameters to be applied together with clinical observations in order to diagnose the disease early.

Patients and methods: The skin disorders induced by the disease were studied using noninvasive techniques: Tewameter TM300, Corneometer CM825, Skin-pH-Meter PH900, Mexameter MX16, Visioscan VC98, and Cutometer MPA580. Twenty-four patients divided into young group, WS group, and elderly group were recruited for the study.

Results: The WS skin is quite similar to aged skin, with some differences concerning the barrier function and skin elasticity; for instance, a WS patient of 30 years of age has the same skin roughness of a 50/60 years old subject with a more severe skin condition leading to higher dryness, high transepidermal water loss, and less distensibility correlating with skin indurations. Conclusion: In patients with WS, the biophysical parameters can quantify the damage induced on the skin by the disease. In order to stage the degree of the disease, biophysical parameters could be used in the future as a diagnostic procedure in the initial stages of the disease as they may reveal lesions not yet clinically perceptible or in advanced stages.

Keywords: Werner syndrome, biophysical parameters, skin aging, noninvasive analysis

\section{Introduction}

Werner syndrome (WS) is a rare autosomal recessive disorder that affects connective tissue throughout the body and is characterized by premature aging in adults; the disease has a high prevalence in Sardinia and Japan. The patients are normal until the second to third decade of life, and then develop premature aging. ${ }^{1} \mathrm{WS}$ is caused by a WRN mutation, located on chromosome 8p11-12 encoding one of the 5 human RecQ helicases. ${ }^{2}$ The prevalence of specific WRN mutations varies depending on the level of consanguinity. Heterozygote frequency in Sardinian population is estimated to be of the order of $1 / 120 .{ }^{3}$ WS is a syndrome of genetic instability characterized by a high percentage of chromosomal translocations and mutations and by a high rate of mutations in somatic cells. To date, 19 different mutations have been identified. All mutations, regardless of whether they are point mutations, insertions, or deletions, lead to the formation of a truncated protein. The WRN truncated proteins resulting from the 
various mutations differ in length from 176 to 1,304 amino acids; some contain the helicase domain, while others are constituted only by the $\mathrm{N}$-terminal end of the protein. These mutations in the WRN protein lead to a destabilization of the heterochromatin. ${ }^{4}$ The affected subjects are usually normal during childhood and begin to show some cardinal clinical signs at the beginning of the second or third decade of life: bilateral cataracts, hair graying and thinning, diabetes, skin ulcers, and early onset of others disorders associated with senescence. There are no standard diagnostic criteria, although several have been used in the field of research. Among the few early signs that appear at puberty and can lead to a suspected diagnosis, the following can be observed: a persistent short stature and an alteration of the dentition, but they are not enough to diagnose WS. To date, there are only clinical or histopathological descriptions of this disease, and there is no study in the literature that quantitatively describes the changes induced by the disease on the skin structure through noninvasive techniques. In addition, this work aims at quantifying the signs of WS skin aging through biophysical parameters already used in dermatology research, and therefore to find new parameters to be applied together with clinical observations in order to help in early diagnosis of this disease.

\section{Patients and methods Study design}

This explorative study was conducted at the Skinlab, Department of Biomedical Sciences, University of Sassari, Sassari, Italy, according to the principles of the current version of the Declaration of Helsinki.

Forty-four Sardinian patients, divided into 3 groups were recruited randomly from the Dermatology Unit of Sassari University Medical School.

Group 1 (young group): 20 volunteers (10 female, 10 male; age range, 24-35 years; mean, 29.5 \pm 3.5 years);

Group 2 (WS group): 4 patients ( 2 female, 2 male; age range, 24-33 years; mean, 28.8 \pm 3.7 years);

Group 3 (elderly group): 20 volunteers (10 female, 10 male; age range, 50-60 years; mean, $66.7 \pm 5.5$ years).

All patients with WS presented signs of the 4 main groups of diagnostic Goto et $\mathrm{al}^{5}$ criteria, ie, short stature, beaked nose, thinning hair, osteoporosis, cataracts, atrophic skin, tense skin especially over the extremities of the feet, and diabetes mellitus (Figure 1). All participants in groups 1 and 3 with no apparent signs of skin disease had Fitzpatrick skin type III and did not smoke or had quit smoking 5 years prior to the study, underwent no hormonal replacement therapy in

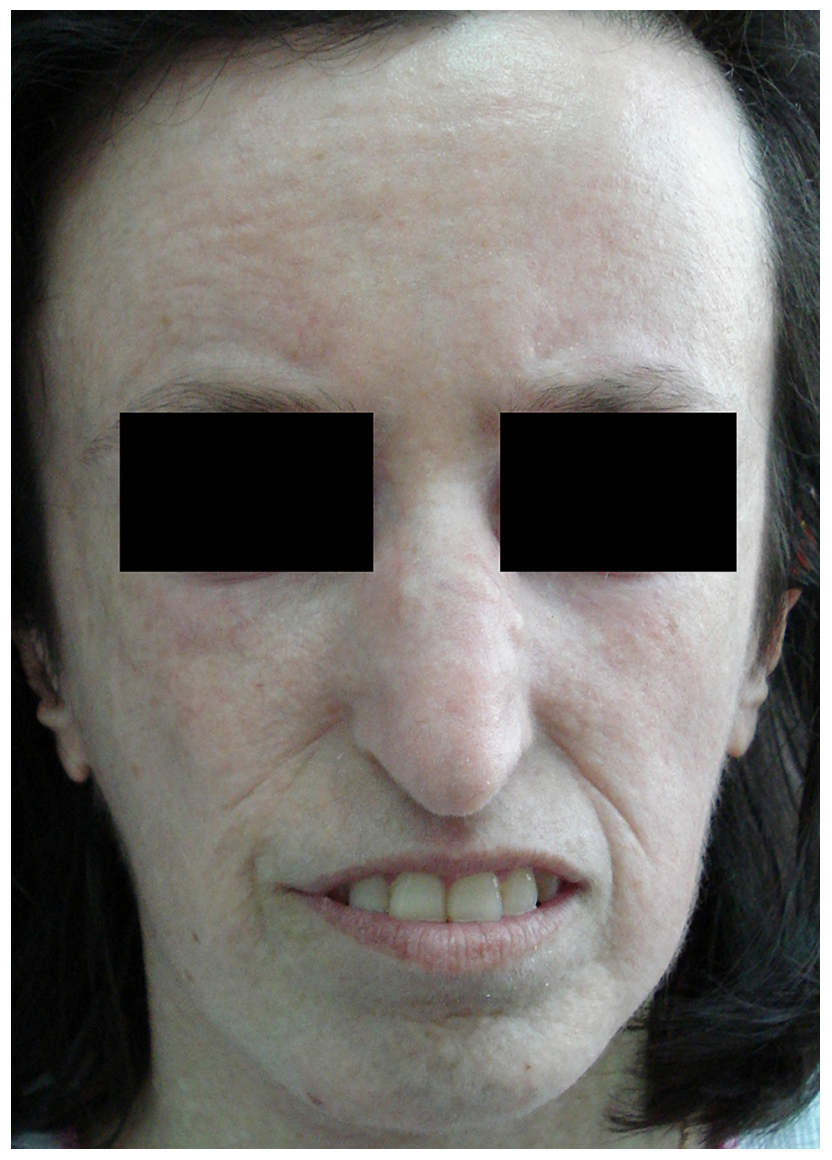

Figure I Woman of 30 years of age with WS. Abbreviation: WS, Werner syndrome.

the previous 2 years, and reported no extreme sun exposure during the 2 months preceding the study. All clinical studies were reviewed and approved by the Ethical Committee Local Health Unit 1 (Sassari, Italy), and written informed consent was obtained from each subject before the study. After meeting the inclusion criteria, informed consent was obtained for the publication of the image.

\section{Biophysical measurements}

The skin disorders induced by the disease were studied using noninvasive techniques. The skin barrier function was evaluated by measurement of the transepidermal water loss (TEWL) using the Tewameter TM 300 (Courage+Khazaka electronic $\mathrm{GmbH}$, Cologne, Germany), hydration in the stratum corneum was assessed using the Corneometer CM 825 (Courage+Khazaka electronic $\mathrm{GmbH}$ ), and skin surface $\mathrm{pH}$ was measured using the Skin-pH-Meter PH 900 (Courage+Khazaka electronic $\mathrm{GmbH}$ ). All measurements were conducted according to current guidelines. ${ }^{6-8}$ The skin color was measured with the Mexameter MX 16 (Courage+Khazaka electronic $\mathrm{GmbH}$ ). A melanin index is 
calculated from the strength of the absorbed and the reflected light at 660 and $880 \mathrm{~nm}$, respectively. An erythema index is computed from the intensity of the absorbed and the reflected light at 568 and $660 \mathrm{~nm}$, respectively. ${ }^{9}$ The skin roughness was evaluated by using the Visioscan VC98, and the software SELS 2000 (Courage+Khazaka electronic GmbH, Cologne, Germany) was used. The device consists of a UVA light source and a charge-coupled device camera, which produces gray scale images of a measurement area sized $6 \times 8 \mathrm{~mm}$. The DIN/ISO parameters maximal roughness (Rmax) and the arithmetic mean roughness from 5 consecutive sampling lengths $(\mathrm{Rz})$ were adopted as previous studies showed high reliability and validity estimates in elderly subjects in these parameters. ${ }^{10}$ The DIN roughness parameters were measured along 100 circularly arranged lines of the measurement field (star roughness). The skin elasticity was measured through a Cutometer MPA 580 and its accompanying software (Courage+Khazaka electronic $\mathrm{GmbH}$ ). A probe opening of $2 \mathrm{~mm}$ diameter and a pressure of $-450 \mathrm{mbar}$ were used. Measurements were performed after 3 subsequent cycles of 2 seconds suction and 2 seconds relaxation. The mechanical properties were expressed as skin extensibility (Uf $=[\mathrm{R} 0])$, skin viscoelasticity (ratio $\mathrm{Uv} / \mathrm{Ue}=[\mathrm{R} 6]$ ), and skin firmness (ratio $\mathrm{Ur} / \mathrm{Uf}=[\mathrm{R} 7])$. Cutometer measurements were performed in duplicate and in accordance to the available guidelines. ${ }^{11}$ All measurements were taken under similar controlled conditions (room temperature of $22^{\circ} \mathrm{C} \pm 3^{\circ} \mathrm{C}$, relative humidity of $55 \% \pm 3 \%$ ) after an acclimatization period of at least 20 minutes, by the same physician. Since the volar forearm is the skin area most often investigated in dermatological research, images and measurement were taken from the subjects' volar forearm, $10 \mathrm{~cm}$ above the crease of the inner wrist. Subjects were instructed to neither wash their volar forearms nor to use any cosmetic products 12 hours prior to measurements.

\section{Statistical analysis}

Statistical analysis was performed using Kruskal-Wallis test for multiple-group comparisons. A post hoc analysis conducted using the Bonferroni correction or paired Mann-Whitney $\mathrm{U}$ tests were performed when Kruskal-Wallis test showed significant differences $(P<0.05)$. Statistical software, SPSS 11.5 for Windows (SPSS Inc., Chicago, IL, USA) was used for analysis with $P<0.05$ considered as statistically significant.

\section{Results}

Means and SDs of the biophysical measurements are shown in Table 1. In our study, the hydration, TEWL, $\mathrm{pH}$, and the colorimetry did not change with aging. The $\mathrm{pH}$, erythema index, and melanin index of the forearm skin in WS patients
Table I Means and standard deviations of biophysical measurements and the statistical difference between the age groups

\begin{tabular}{|c|c|c|c|}
\hline Biophysical parameters & $\begin{array}{c}\text { Young } \\
\text { (24-35 years) }\end{array}$ & Werner & $\begin{array}{c}\text { Elderly } \\
\text { (50-60 years) }\end{array}$ \\
\hline \multicolumn{4}{|c|}{ Skin barrier, mean (SD) } \\
\hline & ** & ** & \\
\hline Corneometry & $40.7\left(7.3_{* \star}\right.$ & $36.6(6.9)_{* *}$ & $39.1(7.2)$ \\
\hline TEWL & $12.1(4.3)$ & $13.9(1.1)$ & $11.4(3.9)$ \\
\hline pH-metry & $5.5(0.6)$ & $5.6(0.7)$ & $5.8(0.6)$ \\
\hline \multicolumn{4}{|c|}{ Skin color, mean (SD) } \\
\hline Erythema index & $586.3(12.2)$ & $583.3(14.8)$ & $577.9(19.4)$ \\
\hline Melanin index & $443.4(10.8)$ & 434.9 (31.3) & $422.5(18.3)$ \\
\hline
\end{tabular}

\begin{tabular}{|c|c|c|c|}
\hline \multicolumn{4}{|c|}{ Skin roughness, mean (SD) } \\
\hline & ** & & \\
\hline \multirow[t]{2}{*}{$\mathrm{R}_{\mathrm{z}}$} & $44.3(8.5)$ & $\underset{\star \star}{52.9}(4.7)$ & $54.4(9.6)$ \\
\hline & \multicolumn{3}{|c|}{ ** } \\
\hline \multirow[t]{4}{*}{$R_{\max }$} & $59.3(8.7)$ & $64.2(5.1)$ & $66.4(9.6)$ \\
\hline & \multicolumn{3}{|c|}{ Skin elasticity, mean (SD) } \\
\hline & \multicolumn{3}{|c|}{$\star * *$} \\
\hline & $* * *$ & $*$ & $\neg$ \\
\hline \multirow[t]{2}{*}{$R_{0}(\mathrm{Uf})$} & $0.91(0.09)$ & $0.44(0.04)$ & $0.71(0.07)$ \\
\hline & $* * *$ & \multicolumn{2}{|l|}{ * } \\
\hline \multirow[t]{2}{*}{$R_{6}(\mathrm{Uv} / \mathrm{Ue})$} & $0.69(0.12)$ & $1.39(1.31)$ & $1.10(0.05)$ \\
\hline & $\star \star \star * *$ & \multicolumn{2}{|l|}{ *** } \\
\hline$R_{7}(\mathrm{Ur} / \mathrm{Uf})$ & $0.47(0.03)$ & $0.73(0.08)$ & $0.39(0.06)$ \\
\hline
\end{tabular}

Notes: $* P \leq 0.05, * * P \leq 0.01, * * * P \leq 0.001$. Corneometry: stratum corneum hydration (arbitrary units); TEWL in $\mathrm{g} / \mathrm{m}^{2} / \mathrm{h} ; R_{z}$ in $\mu \mathrm{m} ; R_{\max }$ in $\mu \mathrm{m} ; R_{0}$ in $\mathrm{mm} ; R_{6}$ is given as a ratio; $R_{7}$ is given as a ratio.

Abbreviations: $R_{0}$, structural extensibility; $R_{6}$, portion of the viscoelasticity; $R_{7}$, elastic recovery; $R_{\text {max }}$ maximal roughness; $R z$, mean depth of roughness; TEWL, transepidermal water loss; Uf, skin distensibility; Uv, skin delayed distension; Ue, skin immediate distension; Ur, skin immediate retraction.

did not show differences compared to other groups. Instead, the hydration and TEWL in WS showed differences in comparison with the other 2 groups. The remaining parameters changed with age. The degree of cutaneous roughness of WS patients was equal to that of elderly healthy subjects, showing no significant differences compared to them. The skin elasticity of WS showed differences compared to the 2 groups enrolled: $R_{0}$ decreases while $R_{6}$ and $R_{7}$ increase.

\section{Discussion}

WS is a progeroid syndrome characterized by early aging signs and symptoms, endocrinologic abnormalities, neoplasms and, scleroderma-like skin changes. The latter show tight and atrophic skin with sclerosis; in addition, ulceration, hyperkeratosis, and pigment alterations of the skin can be seen., ${ }^{3,5,12,13}$ The cutaneous WS skin is also characterized by a decrease in skin structure leading to an epidermal and dermic 
atrophy, reduction of the adipose tissue until disappearance, and reduction of hair follicles as well as sweat and sebaceous glands. Skin changes are the earliest signs that best describe the syndrome, while the most affected areas are the face and the extremities. Moreover, these changes, unlike the other progeria, appear between 15 and 30 years of age. There is still a substantial lack of studies using objective and noninvasive methods which may be helpful in skin assessment of WS patients or those with other progerias. The aim of the present work was to study changes induced on the skin by WS, using noninvasive techniques to understand whether these can quantify the damage and if they might provide future diagnostic support both during early and advanced stages of the disease. The biophysical parameters used aimed to assess the condition of the skin barrier, the color, the elasticity, and the roughness of the anterior surface of the forearm near the wrist. The measurements taken on the forearms are more significant in comparison with the face, where the combination of aging and photoaging increased the variability of the measurements. Most of investigated parameters were associated with chronological age, which is in accordance with previous findings. ${ }^{9,14}$ The WS skin aspect emerging from our studies is quite similar to aged skin, with some differences concerning the barrier function and skin elasticity (Table 1). The skin barrier changes in WS present a less hydrated skin and an increased TEWL compared to the 2 examined groups. The reduced sebaceous and sweat secretion may be the cause of skin dehydration. Another cause might be the presence of skin atrophy that determines a low amount of stratum corneum barrier lipids at the epidermal level, causing, as a consequence, the high level of TEWL, as demonstrated by other authors. ${ }^{15}$ Comparing the 2 control groups, the skin barrier (corneum hydration, TEWL, and $\mathrm{pH}$ ) remained unchanged. Likewise, some other investigations found no relation between forearm skin hydration, ${ }^{16-18} \mathrm{TEWL},{ }^{19-21} \mathrm{pH},{ }^{17}$ and age. The skin color of the forearm in patients with WS does not differ from the other 2 groups; in the present study, also no correlation was found between skin color and age, as shown in other works. ${ }^{14}$ Skin roughness produced an age-dependent increase in $R_{\mathrm{z}}$ and $R_{\max ,}$ in accordance with other studies. ${ }^{22,23}$ Roughening during skin aging might be explained by age-related breakdown of collagen fibers and hyaluronic acid, which is associated with a linear decrease in mechanical strength and dermal water content. At the epidermal level, the increase of roughness might be associated with an increase of heterogeneity of the basal cell size, keratinocytes, and corneocytes. ${ }^{24}$ Additionally, the stratum corneum of aged skin displays a much slower turnover rate,${ }^{25,26}$ possibly contributing to the increase of roughness. In the skin of WS patients, these 2 parameters show significant differences compared to young volunteers but no difference compared to the elderly. Therefore, the degree of cutaneous roughness in the subjects with WS resembles the skin of elderly subjects. The cutaneous elasticity of the forearm in WS has differences when comparing the 2 analyzed groups. Parameter $R_{0}$ was significantly decreased while $R_{6}$ and $R_{7}$ were significantly increased on the volar forearm of the patients with WS compared to healthy controls. The parameters analyzed by Cutometer are related to skin structure and composition, which, in WS subjects, undergo scleroderma-like skin changes. Skin distention $\left(R_{0}\right)$ is linked to the stretching of collagen and elastic fibers and inversely correlates with skin thickness and rigidity. Skin viscoelasticity $\left(R_{6}\right)$ indicates the proportion of viscoelastic plus viscous deformation to elastic deformation of the skin and is attributed to the displacement of the interstitial fluid throughout the fibrous network. It increases in the presence of dermal edema and alterations of interstitial fluid. ${ }^{27}$ Previous studies ${ }^{28,29}$ showed that sclerodermatous skin on the volar forearm was characterized by lower $R_{0}$ and higher $R_{6}$. The parameter $R_{7}$ in sclerodermal skin is significantly reduced in the anterior region of the forearm due to the disappearance of elastic fibers in the sclerotic area. ${ }^{28}$ In our work, $R_{7}$ increased, indicating the presence of elastic fibers in the dermis as evidenced in histological sections, similar to what was observed by other researchers. In all WS patients studied, the suction curve was lower than in the other 2 groups with reduced $U_{\mathrm{e}}$ and $U_{\mathrm{v}}$ values due to the greater rigidity of the dermis as already described by other authors who studied elasticity in patients with scleroderma. ${ }^{28-30}$ These studies demonstrated that the use of skin elasticity meter is reliable and may detect scleroderma or atrophic changes invisible to the naked eye. The $R_{0}$ and $R_{7}$ parameters increase even in the presence of epidermal atrophy alone. ${ }^{30}$

\section{Limits}

This study was conducted on a WS limited sample of 4 individuals due to the rarity of the disease and the refusal of some other patients to undergo new clinical tests. The comparison was made with an additional 20 patients of equal age with healthy skin and 20 elderly with aged skin free of other skin diseases. To give value to the experimentation, we used nonparametric tests with post hoc analysis. There is nothing wrong with conducting well-designed small studies; they just need to be interpreted carefully and data should be used to design larger confirmatory studies. Therefore, this is an exploratory work that has offered some important indications to be explored in further studies. 


\section{Conclusion}

Our work shows that in patients with WS the biophysical parametric can quantify the damage induced on the skin by the disease. The aged appearance of the skin is confirmed by roughness and epidermal and glandular atrophy by corneometry and TEWL, while the dermal damage is assessed through the elasticity parameters. In general, a WS patient of 30 years of age has the same skin roughness of a 50-60 years old subject, with a more severe skin condition leading to higher dryness, high TEWL, and less distensibility, correlating with skin indurations. Based on these results, the biophysical parameters could be used in the future as a diagnostic procedure in the initial stages of the disease as they may reveal lesions not yet clinically perceptible, or they can be used in advanced stages in order to stage the degree of the disease. This study may also provide useful indications in relation to cosmetic dermatology treatments for WS patients. In fact, in daily consultations, it would be advisable to recommend to patients with WS the application of moisturizing products, the use of a mild detergent cleansing product, and the use of antioxidants at a young age.

\section{Disclosure}

The authors report no conflicts of interest in this work.

\section{References}

1. Coppede F. Premature aging syndrome. Adv Exp Med Biol. 2012;724: 317-331.

2. Ishikawa N, Nakamura K, Izumiyama-Shimomura N, et al. Accelerated in vivo epidermal telomere loss in Werner syndrome. Aging. 2001;3(4):417-429.

3. Masala MV, Scapaticci S, Olivieri C, et al. Epidemiology and clinical aspects of Werner's syndrome in North Sardinia: description of a cluster. Eur J Dermatol. 2007;17(3):213-216.

4. Zhang W, Li J, Suzuki K, et al. Aging stem cells. A Werner syndrome stem cell model unveils eterochromatin alterations as a driver of human aging. Science. 2015;348(6239):1160-1163.

5. Goto M, Miller RW, Ishikawa Y, Sugano H. Excess of rare cancers in Werner syndrome (adult progeria). Cancer Epidemiol Biomarkers Prev. 1996;5(4):239-246.

6. Rogiers V. EEMCO guidance for the assessment of transepidermal water loss in cosmetic sciences. Skin Pharmacol Appl Skin Physiol. 2001;14(2):117-128.

7. Myer K, Maibach H. Stratum corneum evaluation methods: overview. Skin Res Technol. 2013;19(3):213-219.

8. Parra JL, Paye M; EEMCO Group. EEMCO guidance for the in vivo assessment of skin surface pH. Skin Pharmacol Appl Skin Physiol. 2003;16(3):188-202.

9. Clarys P,Alewaeters K, Lambrecht R, et al. Skin color measurements: comparison between three instruments: the Chromameter ${ }^{\circledR}$, the Derma Specrometer®, and the Mexameter®. Skin Res Technol. 2000;6(4):230-238.
10. Trojahn C, Schario M, Dobos G, Blume-Peytavi U, Kottner J. Reliability and validity of two in vivo measurements for skin surface topography in aged adults. Skin Res Technol. 2015;21(1):54-60.

11. Rodrigues L; EEMCO. EEMCO guidance to the in vivo assessment of tensile functional properties of the skin. Part 2: instrumentation and test modes. Skin Pharmacol Appl Skin Physiol. 2001;14(1):52-67.

12. Yamamoto K, Imakiire A, Miyagawa N, Kasahara T. A report of two cases of Werner's syndrome and review of the literature. J Orthop Surg. 2003;11(2):224-233.

13. Kyng K, Croteau DL, Bohr VA. Werner syndrome resembles normal aging. Cell Cycle. 2009;8(15):2323.

14. Firooz A, Sadr B, Babakoohi S, et al. Variation of biophysical parameters of the skin with age, gender, and body region. ScientificWorldJournal. 2012;2012:386936.

15. Kolbe L, Kligman AM, Schreiner V, Stoudemayer T. Corticosteroidinduced atrophy and barrier impairment measured by non-invasive methods in human skin. Skin Res Technol. 2001;7(2):73-77.

16. Cua AB, Wilhelm KP, Maibach HI. Frictional properties of human skin: relation to age, sex and anatomical region, stratum corneum hydration and transepidermal water loss. Br J Dermatol. 1990;123(4): 473-479.

17. Wilhelm KP, Cua AB, Maibach HI. Skin aging: effect on transepidermal water loss, stratum corneum hydration, skin surface $\mathrm{pH}$, and casual sebum content. Arch Dermatol. 1991;127(12):1806-1809.

18. Wendling PA, Dell'Acqua G. Skin biophysical properties of a population living in Valais, Switzerland. Skin Res Technol. 2003;9(4):331-338.

19. Roskos KV, Maibach HI, Guy RH. The effect of aging on percutaneous absorption in man. J Pharmacokinet Biopharm. 1989;17(6): 617-630.

20. Tupker RA, Coenraads PJ, Pinnagoda J, Nater JP. Baseline transepidermal water loss (TEWL) as a prediction of susceptibility to sodium lauryl sulphate. Contact Dermatitis. 1989;20(4):265-269.

21. Giusti F, Martella A, Bertoni L, Seidenari S. Skin barrier, hydration, and $\mathrm{pH}$ of the skin of infants under 2 years of age. Pediatr Dermatol. 2001;18(2):93-96.

22. De Paepe K, Lagarde JM, Gall Y, Roseeuw D, Rogiers V. Microrelief of the skin using a light transmission method. Arch Dermatol Res. 2000;292(10):500-510.

23. Fujimura T, Haketa K, Hotta M, Kitahara T. Global and systematic demonstration for the practical usage of a direct in vivo measurement system to evaluate wrinkles. Int J Cosmet Sci. 2007;29(6):423-436.

24. Yaar M. Clinical and histological features of intrinsic versus extrinsic skin aging. In: Gilchrest BA, Krutmann J editors. Skin Aging. Berlin: Springer; 2006:9-22.

25. Grove GL, Kligman AM. Age-associated changes in human epidermal cell renewal. J Gerontol. 1983;38(2):137-142.

26. Tronnier H, Wiebusch M, Heinrich U. Change in skin physiological parameters in space - report on and results of the first study on man. Skin Pharmacol Physiol. 2008;21(5):283-292.

27. O'Goshi KI. Suction chamber method for measurement of skin mechanics: the cutometer. In: Serup J, Jemec GBE, Grove G, editors. Handbook of Non-Invasive Methods and the Skin. Boca Raton: CRC Press Taylor \& Francis Group; 2006:579-582.

28. Dobrev HP. In vivo study of skin mechanical properties in patients with systemic sclerosis. J Am Acad Dermatol. 1999;40(3):436-442.

29. Kalis B, De Rigal J, Leonard F et al. In vivo study of scleroderma by non-invasive techniques. Br J Dermatol. 1990;122(6):457-460.

30. Stamatas GN, Lopes-DaCunha A, Nkengne A, et al. Biophysical properties of Striae Distensae evaluated in vivo using non-invasive assays. Skin Res Technol. 2015;21:254-258. 


\section{Publish your work in this journal}

Clinical, Cosmetic and Investigational Dermatology is an international, peer-reviewed, open access, online journal that focuses on the latest clinical and experimental research in all aspects of skin disease and cosmetic interventions. This journal is included on PubMed. The manuscript management system is completely online and includes a very quick and fair peer-review system, which is all easy to use. Visit http://www.dovepress.com/testimonials.php to read real quotes from published authors 